\title{
latrogenic Airway Foreign Body. A Case Study. - CUERPO EXTRAÑO EN VÍA AÉREA DE ORIGEN IATROGÉNICO. ESTUDIO DE UN CASO.
}

Authors:

Submitted:

Published:

Volume:

Issue:

Affiliation:

Languages:

Keywords:

DOI:

Abstract:

Fabián Gustavo F. Díaz Rodriguez, Hector Julio Melendez Florez 11. August 2018

21. August 2018

5

6

UNIVERSIDD INDUSTRIAL DE SANTANDER

Spanish, Castilian

Anestesia, Cuerpo Extraño, Vía Aérea, Complicación, latrogenia, Intercambiador de tubo. Anesthesia, Foreing body, Airway, Complication, Tube exchanger.

10.17160/josha.5.6.453

We present the case of a 15 years old female patient admitted to the Critical Care Unit. The patient was scheduled for extubation with a tube exchanger. However, the tube exchanger broke inside the patient's airway immediately after extubation requiring surgical intervention, which was successfully performed. 


\title{
IATROGENIC AIRWAY FOREIGN BODY. A CASE STUDY. CUERPO EXTRAÑO EN VÍA AÉREA DE ORIGEN IATROGÉNICO. ESTUDIO DE UN CASO.
}

\author{
AUTORES \\ Díaz Rodríguez Fabián Gustavo (1) \\ Meléndez Flórez Héctor Julio (2) \\ Unidad de Cuidados Intensivos, Hospital Universitario de Santander, \\ Bucaramanga, Colombia.
}

(1) Médico Residente de tercer año, Posgrado Anestesiología y Reanimación, Universidad Industrial de Santander, Bucaramanga, Colombia.

Teléfono: +573016286140

Correo electrónico: fbndzr@gmail.com

(2) Especialista en Anestesiología y Reanimación, MSc Epidemiología, Intensivista, Profesor Titular, Escuela de Medicina, Universidad Industrial de

Santander, Bucaramanga, Colombia.

Teléfono: +573004941492

Correo electrónico: hjmelendez@uis.edu.co

FINANCIACION:

Los autores no han recibido ningún tipo de ayuda o beca durante el desarrollo de dicho manuscrito.

CONFLICTO DE INTERESES:

Los autores declaran no tener ningún conflicto de intereses

RESUMEN

Presentamos el caso de una paciente femenina de 15 años de edad hospitalizada en la Unidad de Cuidado Crítico a quien por antecedente de manipulación quirúrgica prolongada de la vía aérea, se le realizo extubación programada con un intercambiador de tubo, el cual se fracturó dentro de la vía aérea de la paciente inmediatamente después de la extubación.

PALABRAS CLAVE:

Anestesia, Cuerpo Extraño, Vía Aérea, Complicación, latrogenia, Intercambiador de tubo. 
ABSTRACT

We present the case of a 15 years old female patien admited in the Critical Care Unit whose airway was surgically managed, reason why the patient was scheduled for extubation with a tube exchanger, the tube exchanger broke inside the patient's airway inmediatly after extubation.

KEYWORDS: Anesthesia, Foreing body, Airway, Complication, Tube exchanger.

\section{ESTUDIO DE CASO}

Paciente femenina de 15 años de edad con historia de infecciones respiratorias a repetición. Fue admitida en el servicio de urgencias con clínica de 15 días de fiebre, tos con expectoración mucosa no hemoptoica, disnea y dolor pleurítico en hemitorax derecho. Se inicio tratamiento antibiótico para neumonía adquirida en la comunidad, sin necesidad de soporte hemodinámico ni ventilatorio.

El estudio tomográfico contrastado evidencio un tumor endobronquial en el lóbulo medio derecho, neumonía basal derecha y derrame pleural tabicado ipsilateral. La paciente fue programada para biopsia por fibrobroncoscopía la cual reportó histología e inmunohistoquímica compatibles con tumor glómico, la patología de líquido pleural no tuvo evidencia de malignidad.

En junta de neumología se consideró cuadro de obstrucción crónica bronquial por tumor endoluminal que condicionó un estado de atelectasia crónica basal derecha y era la causante de infecciones respiratorias a repetición.

La paciente fue referida al servicio de Cirugía de Tórax; fue llevada a quirófanos en el día 20 de hospitalización. Bajo anestesia general balanceada con tubo bilumen selectivo izquierdo, se realizó toracotomía posterolateral derecha, liberación de adherencias pleurales, decorticación, lobectomía inferior y media, y toracostomía cerrada derecha; posteriormente la paciente fue admitida en la Unidad de Cuidado Crítico (UCl) en el postoperatorio inmediato con analgesia peridural y con intubación orotraqueal con tubo monolumen numero 7.0; a las 24 horas del postoperatorio inmediato la paciente presentó cuadro clínico de desaturación $(50 \%)$, cianosis, y presiones altas de la vía aérea por lo que se fue trasladada a quirófanos donde fue reintervenida quirúrgicamente de urgencia; se realizó re toracotomía y drenaje de escaso hemotórax derecho, posteriormente se evaluó la vía aérea con video-broncoscopio flexible, con hallazgo de un gran coágulo sanguíneo en vía aérea inferior que ocupaba el 95\% de la luz traqueal (tercio distal de tráquea y bronquio fuente derecho) (FIGURAS 1A - imagen propiedad del autor - Visión a través de fibrobroncoscopía rígida, Tráquea obstruida por coagulo y 1B - imagen propiedad del autor - A: bronquio fuente izquierdo, B: bronquio fuente derecho obstruido por coágulo); el coágulo fue de difícil extracción y requirió manipulación de la vía aérea con broncoscopio rígido durante 7.5 horas con participación en conjunto de los servicios de neumología y cirugía de tórax. 
La paciente re ingresó a $\mathrm{UCl}$ con tubo orotraqueal 7.0; se continuo tratamiento antibiótico, sedación, analgesia, nutrición en metas y soporte con ventilación mecánica y vasopresor; al lograr destete de parámetros ventilatorios al sexto día de la re intervención quirúrgica de urgencia, se considero realizar extubación programada con intercambiador de tubo, por sospecha de edema glótico dado la prolongada manipulación de la vía aérea y la alta probabilidad de requerir re intubación orotraqueal con probable vía aérea difícil secundaria al edema sospechado. Al retirar el tubo orotraqueal a través del intercambiador de tubo, inmediatamente el intercambiador se fracturó y la paciente expulsó parte del intercambiador por la cavidad oral quedando parte del dispositivo dentro de la vía aérea, no hubo compromiso en la oxigenación ni en la ventilación de la paciente además de sialorrea y tos ocasional con abundantes secreciones, fue llevada a tomografía de tórax control sin contraste, que evidencio parte el intercambiador de tubo en tráquea y bronquio fuente derecho hasta el sitio del muñón quirúrgico (FIGURA 2 - imagen propiedad del autor - Reconstrucción tridimensional de tomografía de tórax posterior a fractura del intercambiador de tubo. Se observan: A: catéter venoso central subclavio derecho. B: Parte del intercambiador de tubo en la vía aérea. C: Sonda de alimentación nasoyeyunal). Antes de ser intervenida para extraer el cuerpo extraño la paciente expulsó espontáneamente los fragmentos del intercambiador de tubo (FIGURA 3 - imagenes propiedad del autor $-:$ fragmentos del intercambiador dentro de la vía aérea que fueron expulsados espontáneamente por la paciente. C. Fragmento externo del intercambiador de tubo.) sin secuelas ni repercusión en la oxigenación ni en la mecánica ventilatoria, posteriormente con mejoría de la sialorrea y la expectoración. Se tomaron controles radiográficos sin evidencia de fragmentos residuales dentro del tórax ni la vía aérea de la paciente ni complicaciones adicionales durante su estancia hospitalaria.

\section{DISCUSIÓN}

El compromiso de la vía aérea por un cuerpo extraño es una de las mayores urgencias en la práctica médica. Los cuerpos extraños causados por dispositivos médicos constituyen una rara pero potencialmente mórbida y mortal complicación, los casos que se han descrito en la literatura médica abarcan una amplia gama de equipos usados para el cuidado de la vía aérea: a) manguera del spray de anestésico local con el fragmento alojado en el bronquio fuente derecho ${ }^{1}, \mathrm{~b}$ ) componentes del sistema de aspiración de secreciones a través del tubo orotraqueal $^{2}$, c) esfera metálica terminal de un estilete de intubación en paciente pediátrico ${ }^{2}$, d) fractura de un tubo de Montgomery $^{3}$. e) Punta de la funda de un nasofaringoscopio ${ }^{4}$. f) cánula nasofaríngea ${ }^{5}$.g) partes de un broncoscopio ${ }^{6}$ h) cánula metálica de traqueostomía ${ }^{7}$. En la búsqueda bibliográfica realizada sólo se encontró un caso de ruptura de intercambiador de tubo, reportado por Hsien-Yi Lo y colaboradores ${ }^{8}$.

En general los cuerpos extraños en la vía aérea son comúnmente reportados en la población pediátrica ${ }^{9}$. En el caso que reportamos se fracturó el intercambiador de tubo posiblemente por deterioro del material y se fracturo al ser flexionado por la 
presión durante la inserción 0 al ser acodado durante la extracción y desplazamiento del tubo orotraqueal a través de este dispositivo, se consideró en la tomografía como estudio de elección en esta paciente por no existir compromiso en la ventilación ni la oxigenación que ocasionara un manejo urgente a ciegas de esta complicación y se quiso evitar la manipulación innecesaria en el contexto del postoperatorio mediato de la resección pulmonar, de no haber sido expulsados los fragmentos del intercambiador de tubo, la paciente hubiera sido llevada a extracción mediante fibrobroncoscopía flexible ${ }^{10}$.

Advertir la existencia de un cuerpo extraño en vía aérea cuando este no compromete la ventilación ni la oxigenación es difícil, se debe tener una alta sospecha si el caso que se nos presenta no fue presenciado por ningún testigo, el diagnostico inicia con estudios radiológicos simples si tenemos en cuenta que la mayoría de estos objetos son radiolúcidos ${ }^{10}$ por lo que se recomienda tomar rayos $\mathrm{X}$ lateral de tejidos blandos del cuello y placas posterioranterior y lateral del tórax. Es importante recordar que estas imágenes pueden ser normales en las primeras 24 horas. Los exámenes invasivos como la fibrobroncoscopía rígida ó flexible terminan siendo necesarios para tener un diagnostico certero y como parte del tratamiento y extracción del cuerpo extraño ${ }^{11}$.

En el contexto del caso presentado recomendamos la revisión sistemática de los dispositivos médicos antes de ser usados en el cuidado de la vía aérea y la inspección posterior al uso para asegurar que la totalidad del dispositivo haya sido retirado. 


\section{BIBLIOGRAFÍA}

1. Hard C, Nargozian CD. latrogenic airway foreign body. The Journal of the American Society of Anesthesiologists. 1984 Dec 1;61(6):786.

2. Mohnssen SR, Greggs D. latrogenic aspiration of components of respiratory care equipment. CHEST Journal. 1993 Mar 1;103(3):964-5.

3. Qureshi SS, Chaukar D, Dcruz A. Fractured tracheostomy tube in the tracheo-bronchial tree. Journal of the College of Physicians and Surgeons-Pakistan: JCPSP. 2006 Apr;16(4):303-4.

4. Awad Z, Pothier DD. A potential danger of flexible endoscopy sheaths: a detached tip and how to retrieve it. The Journal of Laryngology \& Otology. 2009 Feb 1;123(02):243-4.

5. Dhar V, Al-Reefy H, Dilkes M. Case report-An iatrogenic foreign body in the airway. International Journal of Surgery. 2008 Dec 31;6(6):e46-7.

6. Roach JM, Ripple G, Dillard TA. Inadvertent loss of bronchoscopy instruments in the tracheobronchial tree. CHEST Journal. 1992 Feb 1;101(2):568-9.

7. Myatt JK, Willatts DG. An inhaled tracheostomy tube. Anaesthesia. 1984 Dec 1;39(12):1235-6.

8. Lo HY, Chang KL, Hsieh RY, Tsai TL, Chung WS. An Unusual Tracheobronchial latrogenic Foreign Body-Airway Exchange Catheter. 醫學 與健康期刊. 2016 Mar 1;5(1):125-9.

9. Wu J, Gu M, Wang Z, Li X. A clinical analysis of 21 cases of pen sheath bronchial foreign bodies in children. International journal of clinical and experimental medicine. 2015;8(1):1108.

10.Lemberg PS, Darrow DH, Holinger LD. Aerodigestive tract foreign bodies in the older child and adolescent. Annals of Otology, Rhinology \& Laryngology. 1996 Apr 1;105(4):267-71.

11.Dikensoy O, Usalan C, Filiz A. Foreign body aspiration: clinical utility of flexible bronchoscopy. Postgraduate medical journal. 2002 Jul $1 ; 78(921): 399-403$. 
FIGURA 1A

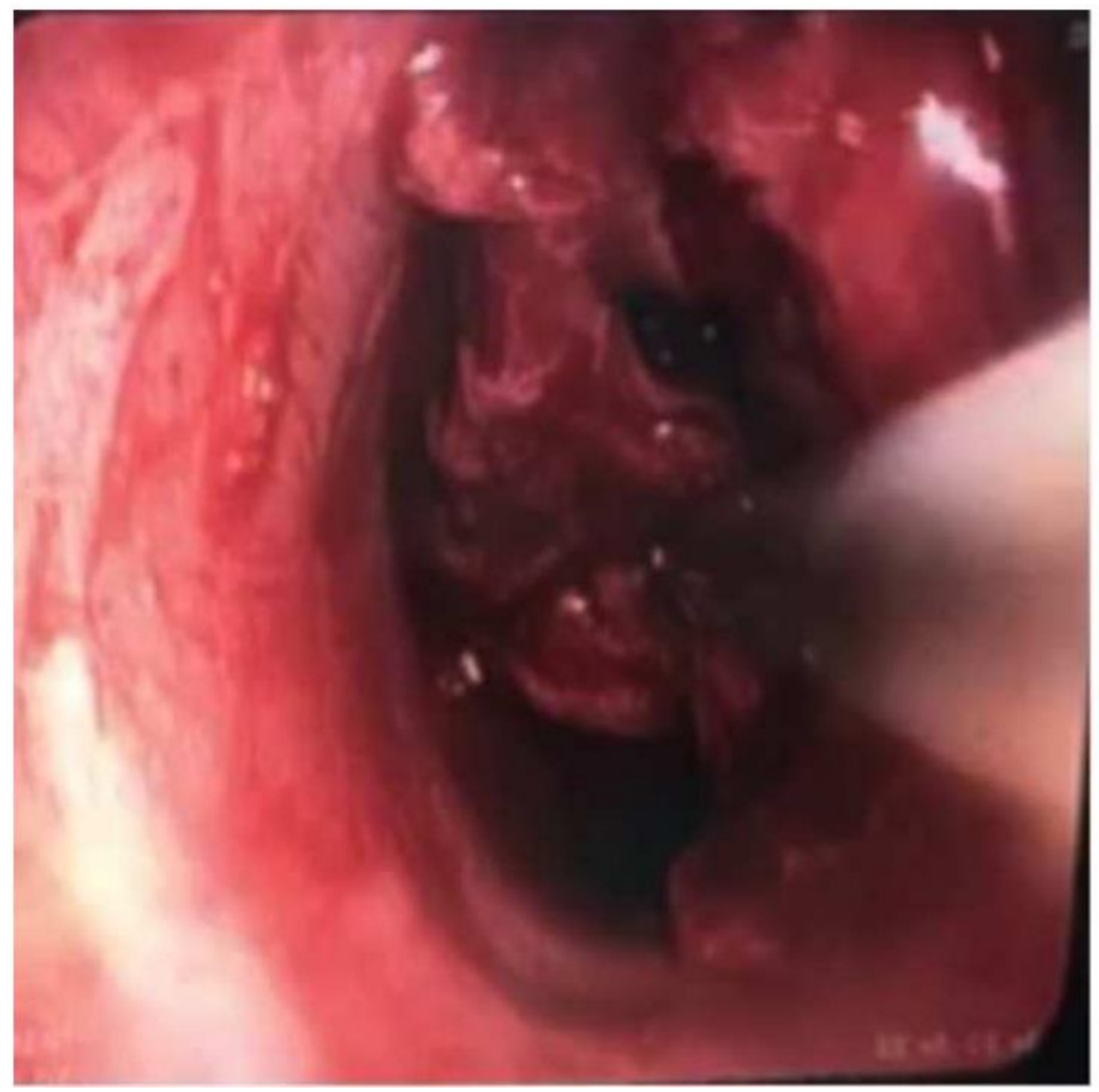


FIGURA 1B

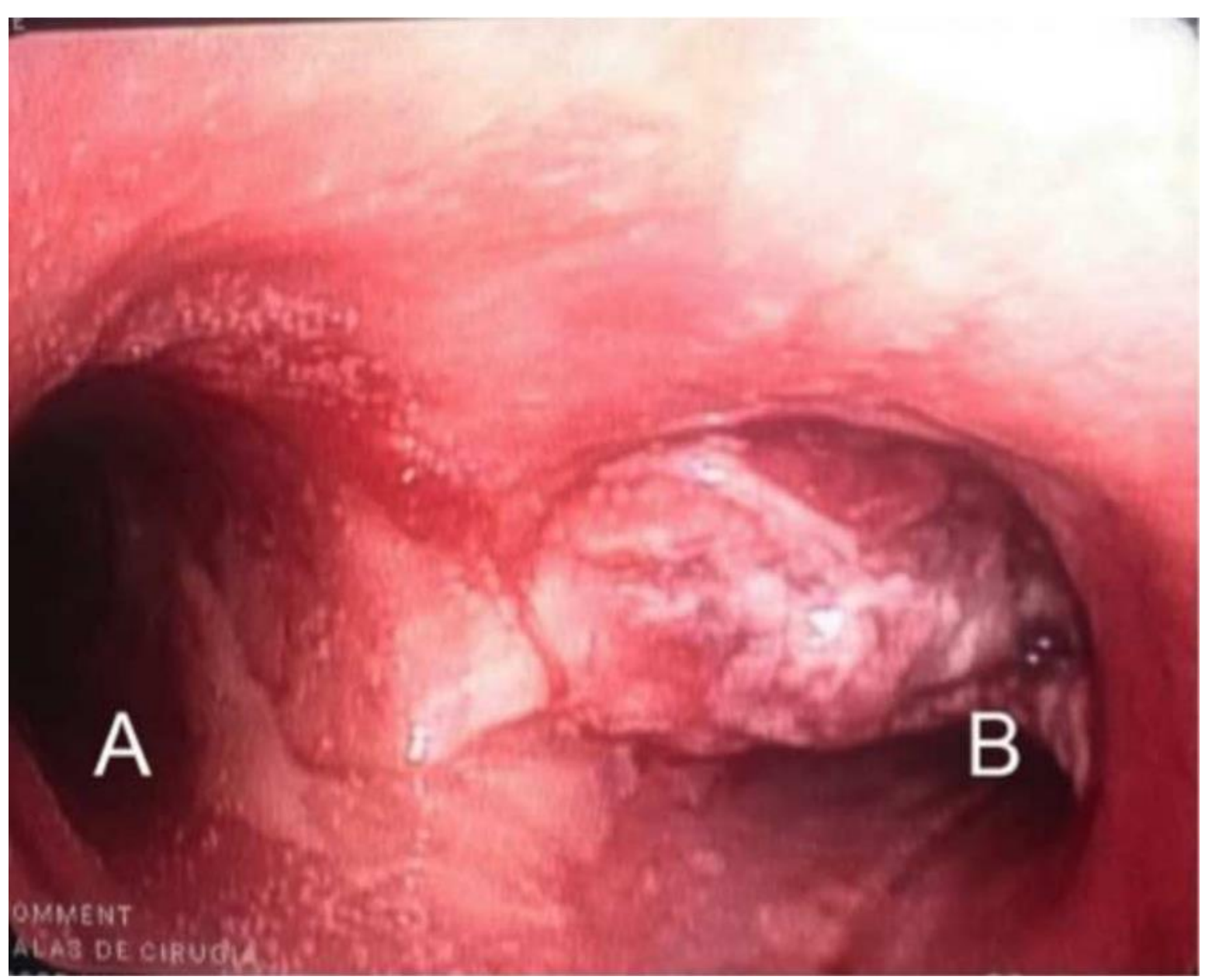


FIGURA 2

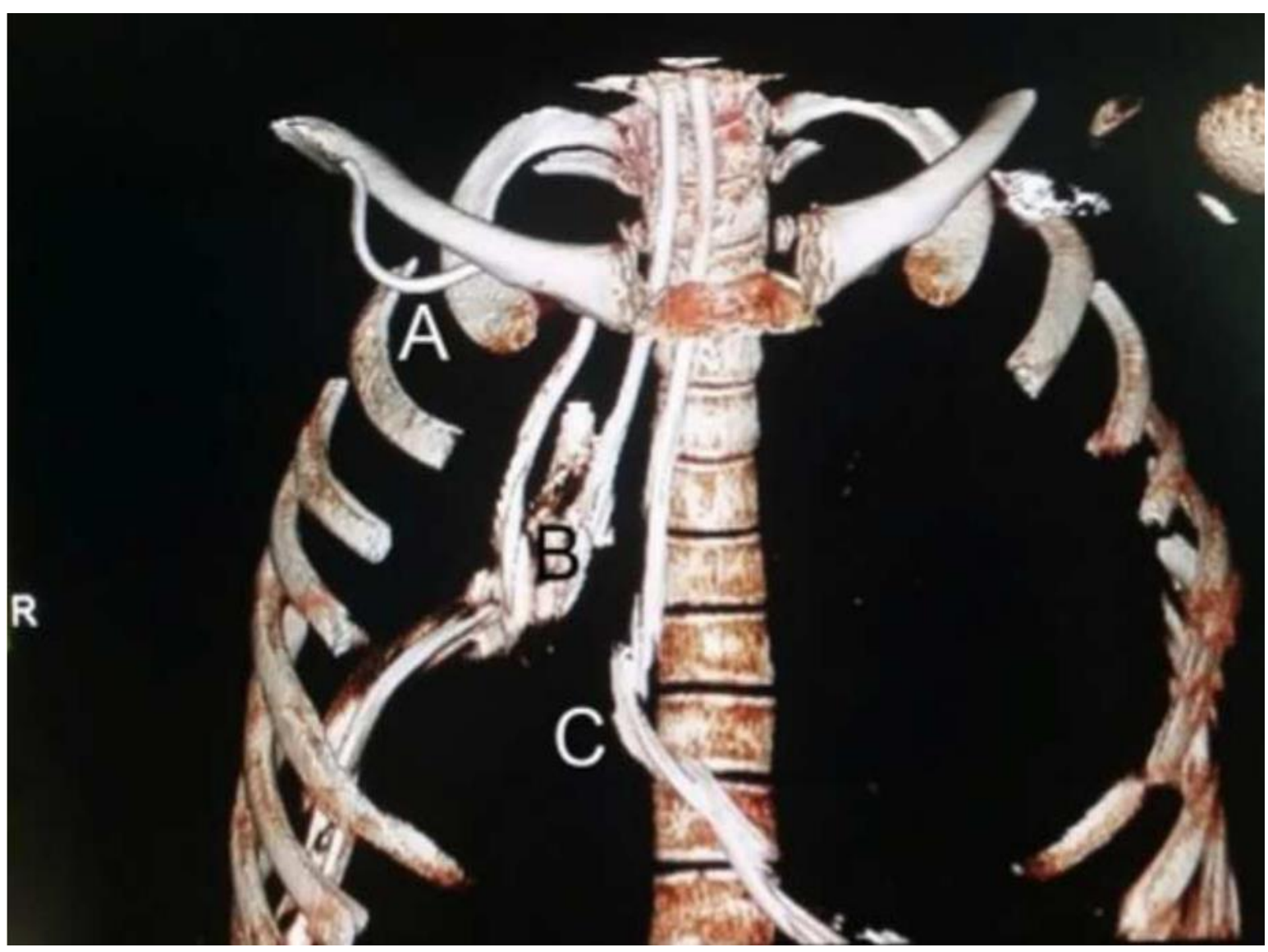


FIGURA 3

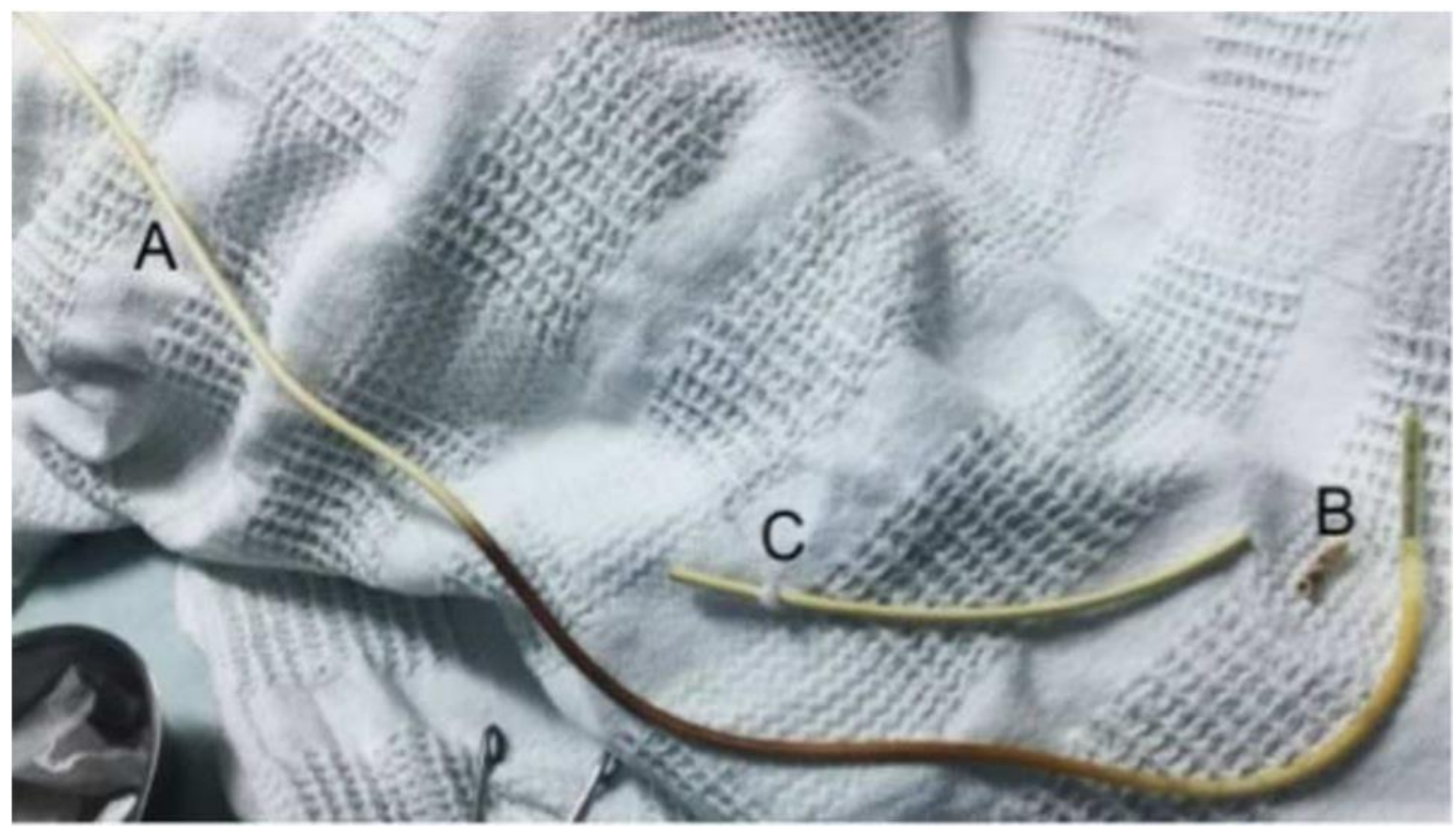




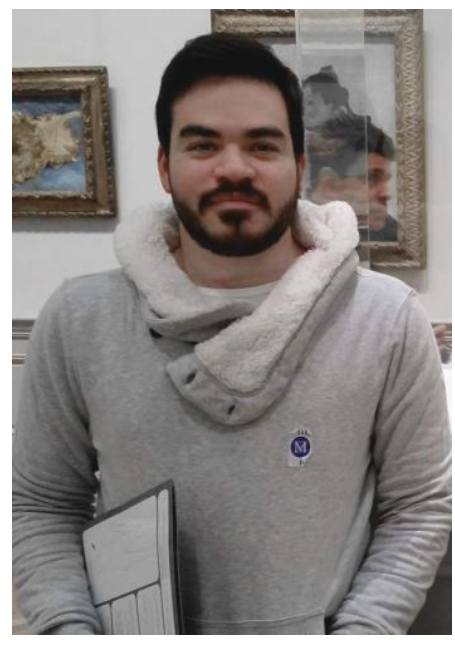

Fabián Gustavo Díaz Rodríguez obtuvo el título de Médico y Cirujano en el año 2011 en la Universidad Industrial de Santander y culminó estudios de especialización en Anestesiología y Reanimación en febrero de 2018 en la misma universidad.

Tiene gran interés en la algología, y en el uso de la ultrasonografía en anestesiología y en cuidados intensivos. 